\title{
The role of databases in drug postmarketing surveillance ${ }^{\dagger}$
}

\author{
Evelyn M. Rodriguez MD, MPH*, Judy A. Staffa PhD, RPh \\ and David J. Graham MD, MPH \\ Office of Postmarketing Drug Risk Assessment, Center for Drug Evaluation and Research, Food \\ and Drug Administration, USA
}

\section{SUMMARY}

This paper describes the role of databases used for postmarketing surveillance of drugs at the United States Food and Drug Administration (FDA). First we describe the Adverse Event Reporting System (AERS), the largest database of adverse event reports in the world. Next, we explain the methods we have used for assembling these adverse event reports into a case series and analysing them, as well as techniques for employing drug use databases to construct reporting rates in the evaluation of drug safety issues. Finally, we discuss the FDA's use of the databases it accesses through its Cooperative Agreement Program to conduct high priority studies to support regulatory decision-making. Published in 2001 by John Wiley \& Sons, Ltd.

KEY WORDS - pharmacoepidemiology; adverse drug reactions; clinical trials, phase IV; consumer product safety; pharmacovigilance

\section{INTRODUCTION}

The Adverse Event Reporting System (AERS) is a relational database that was implemented in November 1997 to replace the Spontaneous Reporting System (SRS), the first database of adverse events reports of the United States Food and Drug Administration (FDA). The SRS, which was started in 1969, is a database collection of all paper images of adverse events reports received by the FDA. In contrast, AERS is a longitudinal database where users can relate one report to subsequent follow-up reports to compile a single case. In addition, AERS technology allows users to encode and search reports using MedDRA (Medical Dictionary for Regulatory Activities Terminology) and to export data into spreadsheets and other computer software for more complex analyses. AERS is an important resource to the FDA and to industry,

\footnotetext{
This article is a U.S. Government work and is in the public domain in the USA.

* Correspondence to: Evelyn M. Rodriguez MD, MPH, Division of Drug Risk Evaluation II, Office of Postmarketing Drug Risk Assessment, Center for Drug Evaluation and Research, FDA, 5600 Fishers Lane, HFD-440, Rockville, MD 20857, USA. Tel: 301-8273172. Fax: 301-480-0628. E-mail: rodrigueze@cder.fda.gov.
}

and has been designed to accept electronic adverse event reports in compliance with the International Conference of Harmonization.

The increased number of drugs on the market and an ever-increasing number of adverse event reports to the FDA have also increased the importance of the efficiencies in data searching and handling offered by AERS. In 1985, FDA received about 50000 reports per year, versus more than 250000 reports in 1999. The robustness of AERS enables the frontline safety evaluation staff to analyse these reports more easily and facilitates their manipulation in other formats.

Seven general principles are considered in evaluating spontaneous reports of adverse events received by FDA. (1) There must be an appropriate temporal relationship such that drug exposure precedes the adverse event. (2) We consider the relationship between the underlying disease, the drug exposure, and the reported adverse event. For example, the exhaustive case analysis for cisapride identified a problem with other drugs that inhibited the cytochrome P450 3A4 enzymes, the same enzymes that metabolize cisapride, or drugs that depleted serum electrolytes. Similarly, thorough case analysis for trovafloxacin revealed that the risk of liver failure 
increased with increasing duration of treatment and led to the withdrawal of the indication for the treatment of chronic prostatitis. (3) We examine the adverse event with respect to concomitant drug use and its relationship to the purported drug exposure and outcome. (4) We consider the patient's medical findings with respect to the current reaction, that is, the clinical onset time, the clinical progression of disease, and confirmation of the diagnosis of the drug reaction. For example, when evaluating reports of severe liver disease we use information regarding pre-existing medical conditions, the results of hepatitis serologic tests, liver biopsies, and autopsy results to make a clinical assessment. (5) We characterize the information derived from drug dechallenge and re-challenge. Whereas de-challenge phenomenon is the resolution of the adverse event when the drug is stopped, re-challenge phenomenon is characterized by recurrence of the adverse event when the person is subsequently re-exposed to the drug. For example, in the cisapride analysis both dechallenge and re-challenge phenomena was exhibited when a patient whose Torsades de Pointe resolved with drug discontinuation and recurred with its readministration while on cardiac monitoring in the cardiac care unit. (6) We consider the feasibility of the drug-adverse event relationship, i.e. is there a biologically plausible relationship between the drug exposure and the adverse event reported? For example, we determine whether pre-clinical studies, especially the animal data, show consistency with the reported adverse event. (7) Finally, we examine known drug class effect relationships with the reported adverse event.

Agency experts responsible for reviewing cases and establishing a case series are safety evaluators who are trained in pharmacy. They partner closely with epidemiologists to evaluate each of the cases, and to develop case definitions for possible and probable cases of adverse drug events. The team also examines the conditions of exposure: duration, dose, timing, and all other characteristics relating to exposure to the drug itself. This process comprises a careful clinical review of each of the individual cases, including potential risk factors or confounding factors for the reported adverse event. The result of this process is a case series of individual events possibly or probably attributed to the drug.

\section{LIMITATIONS OF SPONTANEOUS REPORT DATA}

From a population perspective, case report data provide descriptive information but are generally not use- ful for estimating incidence rates, because of underreporting and the absence of a denominator. The rate of underreporting can be substantial, and might vary with the particular adverse event. There is evidence indicating that the reported cases might represent only $1 \%$ of true cases. ${ }^{1-3}$ The causes of underreporting are multifactorial. More reports are generally received during the first 2 years of a drug's marketing, followed by a gradual decrease thereafter. ${ }^{4}$ The larger pharmaceutical companies with a welldeveloped safety infrastructure and an experienced staff of individuals dedicated to adverse event reporting might generate more reports of higher quality than will be generated by smaller pharmaceutical companies. As noted earlier, there has been a trend toward increased reporting overall in the last 15-20 years. In addition, more reports are received by the FDA when the media brings attention to a particular drug safety issue. Direct-to-consumer advertisements also warn the public of potential adverse events that have been associated with the drug, and it is possible that this might increase reporting from both the public and prescribers as well.

\section{BUILDING A CONTEXT FOR SPONTANEOUS REPORT DATA}

To evaluate spontaneous reports in the proper context of drug use or exposure, reporting rates are calculated to crudely quantify the relationship between the numbers of adverse event reports received by the FDA and the extent of drug use in the US population. Reporting rates consist of a numerator derived only from US spontaneous adverse event reports. The denominator should represent the population at risk for the adverse events, and is estimated from available drug use data with adjustments to estimate the total number of patients exposed to the drug. However, in reality this basic information (e.g. the number of patients in the US who have used the drug) is not readily available. The fragmented nature of the health care system and the mobility of the population in the US make the generation of patient-based national drug use estimates a more formidable task than it might appear at first glance. Furthermore, reporting rates are not incidence rates; not all cases of known adverse events are reported to the FDA, nor do we have the precise number of exposed patients.

Currently the FDA uses data from IMS HEALTH, Inc. to estimate the denominator for reporting rates. These data are derived from two ongoing, crosssectional surveys-the National Prescription Audit PLUS (NPA PLUS) and the National Disease and 
Therapeutic Index (NDTI) - neither of which provides patient-based estimates. NPA PLUS data on dispensed prescriptions come from a panel of approximately 20000 pharmacies across the US (IMS HEALTH, personal communication). IMS projects from this panel to approximate the number of prescriptions filled nationally for any determined time-frame. NDTI is a sample-based survey of approximately 3000 primary care physicians in office-based practice who primarily diagnose and treat disease, to collect data on patient visits (IMS HEALTH, personal communication). Along with patient demographics and diagnoses, the survey collects information about drugs mentioned during the visit. The frequency of these 'mentions' is projected to the universe from which these physicians were sampled. Although these projections cannot be interpreted as the number of patients exposed to a particular drug, they can be used to characterize drug exposure with regard to patient age, sex, and race. Used together, these two data sources can quickly provide a crude picture of the context of drug exposure in which to evaluate spontaneous reports.

For example, the FDA calculated reporting rates for acute liver failure associated with troglitazone using these data. Part of the FDA's independent benefit risk assessment included looking at reporting rates of acute liver failure for troglitazone compared with the other drugs in its class $(107,10.8$, and 13 cases per million person-years for troglitazone, rosiglitazone, and pioglitazone, respectively). These rates were compared with the estimated background incidence rate of acute liver failure in the US of about 1 per million persons per year. ${ }^{5}$ Although we compared a reporting rate to an incidence rate, this type of crude comparison suggested that the reported event might be occurring at a higher than expected frequency in the exposed population, and thus warranted further investigation. This is particularly true when the reporting rate is several orders of magnitude higher than the background rate. In another example, the antiandrogens flutamide, bicalutamide, and nilutamide were compared for risk of reported pulmonary toxicity, including interstitial pneumonitis and pulmonary fibrosis. ${ }^{6}$ The ratio of reporting rates to estimated background incidence for flutamide ( 1 case per million) and bicalutamide ( 5 cases per million) were relatively low, but the ratio for nilutamide (303 cases per million) was high in comparison. This analysis led to labeling changes for nilutamide.

Clearly, patient-based drug exposure data that are national in scope would provide more accurate information for risk assessment. Although this would be useful in all patient populations, there is a particular need to better characterize drug use among hospitalized patients, because IMS HEALTH data has two inadequacies: it is only cross-sectional and data are derived from outpatient visits only. Pediatric drug use in hospitals is especially poorly understood but vitally important for proper implementation of FDA's Pediatric Rule. In addition, there is a need for improved data to address chronically used therapies among ambulatory patients. The FDA is currently developing solicitations for databases in all of these areas.

\section{AUTOMATED RESEARCH DATABASES - THE FDA COOPERATIVE AGREEMENT PROGRAM}

Although the AERS database and drug use data provide tools for preliminary analysis of adverse events, from a scientific, public health, and policy standpoint, there is a great need for better quality information upon which to base decisions relating to drug safety. Population-based data sources offer the opportunity to measure adverse event incidence and to estimate relative and absolute risks.

In an effort to move beyond case report data, FDA funds and utilizes a Cooperative Agreement Program in Pharmacoepidemiology, providing populationbased administrative claims and record-linked data from several different health care settings. The current databases in this program funded through September, 2001, include the Harvard Pilgrim Health Plan (a health maintenance organization), United Health Group (a multistate managed care organization), and Tennessee Medicaid from the US; the Saskatchewan provincial health care database from Canada; and the General Practice Research Database from the UK. Each of these resources captures data on outpatient prescription drugs and medical encounters as well as hospitalizations, reimbursable medical procedures, and laboratory testing, for between 1 and 3 million persons on a longitudinal basis. A special database of approximately 4000 persons infected with HIV from a clinic of the Johns Hopkins Medical Center is used to target safety concerns for the anti-retroviral drugs.

Such databases can be useful in a number of different situations. They provide data on patterns of drug usage (age, sex, duration of use), as well as concomitant prescription medications (drug-drug interactions) and medical conditions (risk factor identification). These databases can also be used to conduct formal epidemiological studies focused on specific drugadverse event hypotheses. In this setting, estimation 


\section{KEY POINTS}

- Pharmacoepidemiology databases are used for postmarketing surveillance of drugs at the United States Food and Drug Administration (FDA)

- The Adverse Event Reporting System (AERS), a passive surveillance system of adverse event reports, is the largest database of adverse event reports in the world

- The FDA uses the databases it accesses through its Cooperative Agreement Program to conduct high-priority studies to support regulatory decision-making

- The AERS database is particularly robust for detecting serious, unexpected adverse drug events

- The FDA has access to other sources of population-based, longitudinal data that can be used to more fully understand drug usage patterns in specific instances where an adverse event was identified. The availability of these databases to the FDA is necessary for advancing the public health

of incidence and relative risk are possible. Because of the large size of the general outpatient population databases, they can be used to identify potential cases with an outcome of interest for purposes of a casecontrol study or case series. The longitudinal nature of all of these databases makes them well suited for studying the effect of FDA regulatory interventions intended to improve the risk-benefit profile and foster the safer use of medicines.

\section{CONCLUSION}

The FDA uses every available source of data to assess drug safety. The AERS database is a passive surveillance system of adverse event reports that is particularly robust for detecting serious, unexpected adverse drug events. Detailed analyses of the literature might yield further evidence for a suspected drug safety signal. Once a signal is identified, the FDA might conduct epidemiologic studies to estimate incidence rates or define risk factors in the evaluation of the suspected drug signal. These ongoing data analyses are critical to the ongoing benefit-risk assessments for drugs once they are marketed in the US.

The FDA has access to other sources of populationbased, longitudinal data that can be used to more fully understand drug usage patterns in specific instances where an adverse event was identified. The availability of these databases to the FDA is necessary for advancing the public health.

\section{REFERENCES}

1. Rogers AS, Israel E, Smith CR, et al. Physician knowledge, attitudes, and behavior related to reporting adverse drug events. Arch Intern Med 1988; 148: 1596-1600.

2. Jorup-Ronstrom C, Britton S. Efficacy of reporting systems of adverse reactions to drugs and care. Scand J Soc Med 1983; 11: 87-89.

3. Scott HD, Rosenbaum SE, Waters WJ, et al. Rhode Island physicians reporting of adverse drug reactions. R I Med J 1987; 70: 311-316.

4. Weber JCP. Epidemiology of adverse reactions to nonsteroidal antiinflammatory drugs. In Advances in Inflammation Research, Vol 6, Rainsford KD, Velo GP (eds). Raven Press: New York, 1984; 1-7.

5. Graham DJ, Waller PC, Kurz X. A view from regulatory agencies. In Pharmacoepidemiology (3rd edn), Strom BL (ed.). John Wiley \& Sons Ltd: New York, 2000; 109-24.

6. Ahmad SR, Graham DJ, Toyer DP, et al. Comparison of pulmonary toxicity risks with antiandrogens. Pharmacoepidemiol Drug Safe 2000; 9(Suppl. 1): S121. 